\title{
Contemporary management and outcomes of infective tunnelled haemodialysis catheter-related right atrial thrombi: a case series and literature review
}

Min Sen ${\underline{Y e w^{1}}}^{1}$, MBBS, MRCP, Andrew Michael Weng Meng Leong ${ }^{1}$, MBBS, MRCP

INTRODUCTION Infective haemodialysis catheter-related right atrial thrombus (CRAT) is a complication of tunnelled catheter use. Management recommendations are based mainly on published case series prior to 2011. We report our institution's recent experience in managing infective haemodialysis CRAT and correlate treatment with outcomes.

METHODS We conducted a retrospective analysis of haemodialysis CRAT cases diagnosed on transthoracic echocardiography between 1 January 2011 and 31 December 2017. Clinical outcomes, including mortality at 180 days post diagnosis and thrombus resolution, were traced from electronic medical records.

RESULTS There were 14 cases identified. The median age was 59 (range 47-88) years and 11 (78.6\%) were male. Sepsis was the most common reason for hospitalisation (71.4\%). Blood cultures identified Staphylococcus aureus in seven cases, of which two were methicillin-resistant. Three had coagulase-negative Staphylococcus. All cases received antibiotics with infectious disease physician input. Seven were treated with catheter removal alone, of which three died within 180 days. Both cases treated with catheter removal plus anticoagulation survived at 180 days. Of the two cases who had anticoagulation without catheter removal, one died within 180 days and the other did not have thrombus resolution. Three underwent surgical thrombus removal, of which two died postoperatively and the last required repeated operations and prolonged hospitalisation. Mortality at 180 days post diagnosis was $42.9 \%$.

CONCLUSION Catheter removal and anticoagulation are modestly effective. Surgery is associated with poor outcomes. Despite contemporary management, infective haemodialysis CRAT still results in high mortality. Prospective studies are needed to identify the optimal management.

Keywords: haemodialysis, infective endocarditis, right atrium, thrombus, tunnelled catheter

\section{INTRODUCTION}

Tunnelled catheters inserted via the internal jugular or subclavian veins are often used to provide vascular access for haemodialysis in end-stage renal failure (ESRF). The National Kidney Foundation recommends positioning of the catheter tip in the right atrium (RA) to achieve better flow rates. ${ }^{(1)}$ However, this may increase the risk of catheter-related right atrial thrombus (CRAT) formation. This is likely due to repeated catheter tip movement against the RA endocardium, resulting in endothelial damage and activation of the coagulation cascade. ${ }^{(2)}$ As bacteraemia can occur frequently during haemodialysis, ${ }^{(3)}$ the formed thrombus serves as a nidus for infection and becomes an infected vegetation. The optimal management for infected haemodialysis CRAT is unknown and can be either one or a combination of parenteral antibiotics, catheter removal, systemic anticoagulation or surgical thrombectomy. The literature available is limited to sporadic case reports and case series published in 2003-2004. ${ }^{(4,5)}$ With improvements in healthcare delivery, diagnostics, medications and surgical techniques, the outcomes for infected haemodialysis CRAT may have changed. We aimed to describe our centre's experience with infected haemodialysis CRAT and correlate treatment modalities with outcomes. A brief review of contemporary case reports published in the literature is also presented.

\section{METHODS}

We retrospectively reviewed all resting transthoracic echocardiography performed in our institution from 1 January 2011 to 31 December 2017 for cases of right atrial thrombus. Only ESRF cases with either a haemodialysis catheter in situ or documented removal of the catheter during the index admission were included. In addition, all included cases must have at least one positive blood culture for a microorganism consistent with infective endocarditis (IE). Information pertaining to patient demographics, presentation, clinical course, investigations, management and outcomes was collected from the hospital's electronic medical records. Shapiro-Wilks test was used to check for normality of data, which was then presented as mean \pm standard deviation, median (range) and proportion, as appropriate. The primary outcome assessed was mortality at 180 days from echocardiographic diagnosis. The secondary outcome was thrombus size seen on follow-up transthoracic echocardiography within 180 days. Ethics approval was obtained from the hospital's institutional review board.

\section{RESULTS}

We identified 14 cases of infective haemodialysis CRAT, and their characteristics are summarised in Table I. The median age of the patients was 59 (range 47-88) years and 11 (78.6\%)

${ }^{1}$ Department of Cardiology, Tan Tock Seng Hospital, Singapore

Correspondence: Dr Yew Min Sen, Consultant, Department of Cardiology, Tan Tock Seng Hospital, 11 Jalan Tan Tock Seng, Singapore 308433. min_sen_yew@ttsh.com.sg 
patients were male. Of the 14 cases, 6 (42.9\%) were known to have ischaemic heart disease previously, including three with a prior myocardial infarction. Diabetes mellitus was the cause of renal failure in all, except one patient who had hypertensive nephrosclerosis. The median duration from catheter insertion to diagnosis of bacteraemia was 92 (range 1-424) days. 12 patients received a Palindrome catheter, with the remaining two cases having a HemoStar® catheter (Bard Access Systems, Inc, Salt Lake City, UT, USA) and a HeRO Graft $($ (Merit Medical Systems, South Jordon, UT, USA), respectively. Venous access was through the right internal jugular in 12 patients (including the case with HeRO Graft), the left internal jugular in one case and the left femoral in the last case. Sepsis was the most common reason for hospitalisation (71.4\%), followed by fluid overload (14.3\%). Blood cultures identified Staphylococcus aureus in 7 (50.0\%) cases, of which $2(28.8 \%)$ were methicillin-resistant. Coagulasenegative Staphylococcus species was the next most commonly isolated organism and was seen in $3(21.4 \%)$ cases. Anaemia, defined as serum haemoglobin $<13 \mathrm{~g} / \mathrm{dL}$ in male patients and $<12 \mathrm{~d} / \mathrm{dL}$ in female patients, ${ }^{(6)}$ was present in all except one patient on admission.

Echocardiographic features of the patients are summarised in Table I. An echocardiographic diagnosis was made after a mean of $6.1 \pm 1.5$ days after admission. The median thrombus size measured on transthoracic echocardiography was 21.5 (range 10-41) $\mathrm{mm}$ by 15.0 (range $6-30$ ) $\mathrm{mm}$. The dialysis catheter could be visualised in 7 (50\%) cases, of which there were adherent thrombi in four cases. The thrombi in the remaining cases were mostly attached to the RA free wall $(n=7)$, followed by the RA wall near the inferior vena cava junction $(n=2)$. Tricuspid annular involvement was seen in two cases, although none of them demonstrated tricuspid leaflet involvement. The left ventricular ejection fraction (LVEF) was less than 50\% in 6 (42.9\%) cases.

All patients received appropriate parenteral antibiotics guided by blood cultures and input from infectious disease physician. The haemodialysis catheter was also removed during the same admission in 12 cases. Three patients had their catheters removed on the day of the blood culture, with the remaining nine catheters removed at a median of 5 (range 1-7) days after blood cultures were taken. One patient (Patient 10) did not have the catheter removed before death (16th day of admission) due to concerns regarding embolic risk in view of the thrombus extent and attachment to the catheter. The patient with the HeRO Graft had surgical graft explantation 325 days after the index admission, as he was initially deemed to be at high surgical risk.

A summary of the various treatment strategies with their corresponding outcomes is presented in Table II. Catheter removal was the sole adjunct to antibiotic therapy, with no anticoagulation or surgery in half the cases. Anticoagulation was used in four patients, of which two did not have catheter removal. Anaemia was cited as the most common reason for not starting anticoagulation (Patients 1, 2, 6 and 8). No documented reason was found for the remaining six patients, although a review of medical records did not reveal any apparent contraindications such as allergies or active bleeding. Three patients underwent surgical removal of thrombus during the index admission, of which two had coronary bypass grafting performed in the same setting. All three patients had documented clearance of bacteraemia by blood cultures before surgery. Of the three, Patient 7 had a preoperative LVEF of $50 \%$, while the other two (Patients 9 and 12) had LVEFs of at least 55\%. Their operative risk, as estimated by the internationally validated EUROSCORE II, ${ }^{(6)}$ was $7.62 \%$ (Patient 7), 2.39\% (Patient 9) and 3.4\% (Patient 12).

The overall mortality at 180 days post echocardiographic diagnosis was $42.9 \%$. A comparison of the characteristics between survivors and non-survivors is presented in Table III. Of the seven patients treated with catheter removal only, 3 (42.9\%) died from sepsis and related complications (such as multiorgan failure) within 180 days of diagnosis. Two survivors had follow-up echocardiography, of which one showed thrombus size reduction and the other demonstrated thrombus resolution. Both patients who received anticoagulation with catheter removal survived at six months, of which one had follow-up imaging that showed complete thrombus resolution. One of the two cases, Patient 10, who received anticoagulation without catheter removal, died from septic shock within 180 days of diagnosis. The remaining patient (Patient 11, who had the HeRO Graft) survived, but the thrombus remained similar in size at 70 days post diagnosis. Out of the three patients who underwent surgery, 2 (66.7\%) died from complications on postoperative day (POD) 32 and 36 , respectively. The last patient (Patient 12) developed cardiac arrest on POD 5 and required repeated operations for wound infection, with prolonged hospitalisation of more than 180 days. There were no cases of pulmonary embolism arising from CRAT, irrespective of the treatment received.

\section{DISCUSSION}

Patients with ESRF who are dependent on tunnelled catheters for haemodialysis are at risk for infective CRAT for multiple reasons. The positioning of the catheter tip at the level of the RA, as recommended by guidelines, ${ }^{(1)}$ may result in RA wall damage and mural thrombus formation due to constant catheter motion against the endocardium. ${ }^{(7)}$ ESRF patients are also inherently at risk of infection due to uraemia-induced immunodepression. ${ }^{(8)}$ Furthermore, haemodialysis is associated with high rates of bacteraemia, ${ }^{(9)}$ particularly if a catheter, rather than an arteriovenous fistula or graft, is used for access. ${ }^{(10)}$

There is limited literature on the optimal management of infective CRAT. Current treatment options consist of either one or a combination of antibiotics, catheter removal, anticoagulation and surgical thrombectomy. Existing therapeutic recommendations for CRAT are based solely on retrospective case series in the last decade, and have either included both infective and sterile CRAT ${ }^{(4,11)}$ or evaluated non-ESRF patients with central catheters. ${ }^{(12)}$ Data on infective CRAT published from 2011 and beyond is limited only to isolated case reports..$^{(2,13-16)}$

Catheter removal is strongly recommended as part of the initial treatment strategy and has been found to influence mortality. ${ }^{(11)}$ In our series, catheter removal alone appears to be modestly effective. Given the potential risk of thrombus 
Case Series

Table I. Demographics, clinical history, presentation and echocardiographic features.

\begin{tabular}{|c|c|c|c|c|c|c|c|c|c|c|c|c|c|}
\hline $\begin{array}{l}\text { Patient } \\
\text { no. }\end{array}$ & $\begin{array}{l}\text { Age (yr)/ } \\
\text { gender }\end{array}$ & $\begin{array}{l}\text { Dialysis } \\
\text { duration } \\
(y r)\end{array}$ & DM & IHD & $\begin{array}{l}\text { Vascular access; } \\
\text { catheter type; } \\
\text { time from } \\
\text { insertion to 1st } \\
\text { positive blood } \\
\text { culture }\end{array}$ & $\begin{array}{l}\text { Clinical } \\
\text { presentation }\end{array}$ & Organism & $\begin{array}{l}\text { Admission } \\
\text { Hb level } \\
\text { (g/dL) }\end{array}$ & $\begin{array}{l}\text { Time from } \\
\text { admission } \\
\text { to echo } \\
\text { (day) }\end{array}$ & $\begin{array}{l}\text { LVEF } \\
\text { (\%) }\end{array}$ & $\begin{array}{l}\text { Thrombus } \\
\text { dimensions } \\
\text { (largest } \times \\
\text { smallest; } \\
\mathrm{mm} \text { ) }\end{array}$ & $\begin{array}{l}\text { Catheter } \\
\text { visualised during } \\
\text { echo }\end{array}$ & $\begin{array}{l}\text { Thrombus } \\
\text { location; } \\
\text { mobility }\end{array}$ \\
\hline 1 & $\begin{array}{l}67 / \\
\text { Male }\end{array}$ & 2 & Yes & Not known & $\begin{array}{l}\text { Right IJ; Bard } \\
\text { 23-cm HemoStar } \\
\text { catheter; } 122 \text { days }\end{array}$ & $\begin{array}{l}\text { Fluid } \\
\text { overload }\end{array}$ & MSSA & 8.4 & 9 & 25 & $19 \times 15$ & Yes & $\begin{array}{l}\text { On catheter; } \\
\text { mobile }\end{array}$ \\
\hline 2 & $\begin{array}{l}55 / \\
\text { Male }\end{array}$ & 1 & Yes & Not known & $\begin{array}{l}\text { Right IJ; } 19-\mathrm{cm} \\
\text { Palindrome } \\
\text { catheter; } 1 \text { day }\end{array}$ & $\begin{array}{l}\text { Fluid } \\
\text { overload }\end{array}$ & MSSA & 7.7 & 6 & 50 & $10 \times 8$ & Yes & $\begin{array}{l}\text { On catheter; } \\
\text { non-mobile }\end{array}$ \\
\hline 3 & $\begin{array}{l}53 / \\
\text { Male }\end{array}$ & 1 & Yes & Not known & $\begin{array}{l}\text { Right IJ; } 23-\mathrm{cm} \\
\text { Palindrome } \\
\text { catheter; } 121 \text { days }\end{array}$ & Sepsis & $\begin{array}{l}\text { CoNS } \\
\text { (Staphylococcus } \\
\text { epidermidis) }\end{array}$ & 8.1 & 5 & 45 & $23 \times 19$ & Yes & $\begin{array}{l}\text { RA free wall and } \\
\text { part of tricuspid } \\
\text { annulus; } \\
\text { some mobile } \\
\text { extensions }\end{array}$ \\
\hline 4 & $\begin{array}{l}58 / \\
\text { Male }\end{array}$ & 1 & Yes & Not known & $\begin{array}{l}\text { Right IJ; } 23-\mathrm{cm} \\
\text { Palindrome } \\
\text { catheter; } 393 \text { days }\end{array}$ & $\begin{array}{l}\text { Cardiac } \\
\text { arrest }\end{array}$ & $\begin{array}{l}\text { Streptococcus } \\
\text { bovis }\end{array}$ & 13.7 & 6 & 25 & $17 \times 14$ & Yes & $\begin{array}{l}\text { RA free wall; } \\
\text { non-mobile }\end{array}$ \\
\hline 5 & $\begin{array}{l}64 / \\
\text { Male }\end{array}$ & 1 & Yes & Not known & $\begin{array}{l}\text { Left IJ; } 28-\mathrm{cm} \\
\text { Palindrome } \\
\text { catheter; } 5 \text { days }\end{array}$ & Sepsis & $\begin{array}{l}\text { Burkholderia } \\
\text { cenocepacia }\end{array}$ & 11.7 & 6 & 60 & $16 \times 11$ & No & $\begin{array}{l}\text { RA wall near } \\
\text { junction with } \\
\text { IVC' } \\
\text { non-mobile }\end{array}$ \\
\hline 6 & $\begin{array}{l}60 / \\
\text { Male }\end{array}$ & 5 & Yes & $\begin{array}{l}\text { Yes; } \\
\text { previous } \\
\text { CABG }\end{array}$ & $\begin{array}{l}\text { Right IJ; 19-cm } \\
\text { Palindrome } \\
\text { catheter; } 105 \text { days }\end{array}$ & Sepsis & $\begin{array}{l}\text { Acinetobacter } \\
\text { baumannii, } \\
\text { Stenotrophomonas } \\
\text { maltophilia }\end{array}$ & 8.5 & 5 & 35 & $27 \times 13$ & No & $\begin{array}{l}\text { RA free wall; } \\
\text { non-mobile }\end{array}$ \\
\hline 7 & $\begin{array}{l}53 / \\
\text { Male }\end{array}$ & 2 & Yes & $\begin{array}{l}\text { Yes; MPI } \\
\text { showed } \\
\text { limited } \\
\text { ischaemia, } \\
\text { treated } \\
\text { medically }\end{array}$ & $\begin{array}{l}\text { Right IJ; } 19-\mathrm{cm} \\
\text { Palindrome } \\
\text { catheter; } 18 \text { days }\end{array}$ & Sepsis & MRSA & 9.9 & 6 & 50 & $30 \times 30$ & No & $\begin{array}{l}\text { RA free wall; } \\
\text { mobile }\end{array}$ \\
\hline 8 & $\begin{array}{l}47 / \\
\text { Female }\end{array}$ & 2 & Yes & Not known & $\begin{array}{l}\text { Right IJ; } 23-\mathrm{cm} \\
\text { Palindrome } \\
\text { catheter; } 30 \text { days }\end{array}$ & $\begin{array}{l}\text { Respiratory } \\
\text { distress }\end{array}$ & MRSA & 7.4 & 6 & 60 & $29 \times 15$ & No & $\begin{array}{l}\text { RA free wall; } \\
\text { mobile }\end{array}$ \\
\hline
\end{tabular}


Case series

Table I. (Contd...)

\begin{tabular}{|c|c|c|c|c|c|c|c|c|c|c|c|c|c|}
\hline $\begin{array}{l}\text { Patient } \\
\text { no. }\end{array}$ & $\begin{array}{l}\text { Age (yr)/ } \\
\text { gender }\end{array}$ & $\begin{array}{l}\text { Dialysis } \\
\text { duration } \\
\text { (yr) }\end{array}$ & DM & IHD & $\begin{array}{l}\text { Vascular access; } \\
\text { catheter type; } \\
\text { time from } \\
\text { insertion to 1st } \\
\text { positive blood } \\
\text { culture }\end{array}$ & $\begin{array}{l}\text { Clinical } \\
\text { presentation }\end{array}$ & Organism & $\begin{array}{l}\text { Admission } \\
\text { Hb level } \\
\text { (g/dL) }\end{array}$ & $\begin{array}{l}\text { Time from } \\
\text { admission } \\
\text { to echo } \\
\text { (day) }\end{array}$ & $\begin{array}{l}\text { LVEF } \\
(\%)\end{array}$ & $\begin{array}{l}\text { Thrombus } \\
\text { dimensions } \\
\text { (largest } \times \\
\text { smallest; } \\
\text { mm) }\end{array}$ & $\begin{array}{l}\text { Catheter } \\
\text { visualised during } \\
\text { echo }\end{array}$ & $\begin{array}{l}\text { Thrombus } \\
\text { location; } \\
\text { mobility }\end{array}$ \\
\hline 9 & $\begin{array}{l}47 / \\
\text { Male }\end{array}$ & 2 & Yes & Not known & $\begin{array}{l}\text { Right IJ; } 23-\mathrm{cm} \\
\text { Palindrome } \\
\text { catheter; } 424 \text { days }\end{array}$ & Sepsis & MSSA & 10.8 & 4 & 60 & $28 \times 27$ & No & $\begin{array}{l}\text { RA free wall and } \\
\text { part of tricuspid } \\
\text { annulus; } \\
\text { non-mobile }\end{array}$ \\
\hline 10 & $\begin{array}{l}60 / \\
\text { Male }\end{array}$ & 1 & Yes & $\begin{array}{l}\text { Yes; } \\
\text { NSTEMI } \\
\text { treated } \\
\text { with PCI } \\
\text { previously }\end{array}$ & $\begin{array}{l}\text { Right IJ; } 23-\mathrm{cm} \\
\text { Palindrome } \\
\text { catheter; } 27 \text { days }\end{array}$ & Sepsis & MSSA & 10.7 & 4 & 20 & $31 \times 26$ & Yes & $\begin{array}{l}\text { On catheter; } \\
\text { non mobile }\end{array}$ \\
\hline 11 & $\begin{array}{l}88 / \\
\text { Male }\end{array}$ & 3 & No & $\begin{array}{l}\text { Yes; } \\
\text { previous } \\
\text { CABG }\end{array}$ & $\begin{array}{l}\text { HeRO Graft; } \\
79 \text { days }\end{array}$ & Sepsis & $\begin{array}{l}\text { Escherichia coli } \\
\text { (pansensitive) }\end{array}$ & 9.2 & 7 & 50 & $18 \times 17$ & Yes & $\begin{array}{l}\text { On catheter; } \\
\text { non-mobile }\end{array}$ \\
\hline 12 & $\begin{array}{l}60 / \\
\text { Male }\end{array}$ & 2 & Yes & $\begin{array}{l}\text { Yes; MPI } \\
\text { showed } \\
\text { small } \\
\text { myocardial } \\
\text { infarct } \\
\text { with no } \\
\text { ischaemia }\end{array}$ & $\begin{array}{l}\text { Right IJ; } 23-\mathrm{cm} \\
\text { Palindrome } \\
\text { catheter; } 152 \text { days }\end{array}$ & Sepsis & $\begin{array}{l}\text { CoNS } \\
\text { (Staphylococcus } \\
\text { capitis) }\end{array}$ & 10.2 & 8 & 55 & $41 \times 28$ & Yes & $\begin{array}{l}\text { Inferior } \\
\text { posterior } \\
\text { RA wall; } \\
\text { some mobile } \\
\text { extensions }\end{array}$ \\
\hline 13 & $\begin{array}{l}\text { 69/ } \\
\text { Female }\end{array}$ & 3 & Yes & $\begin{array}{l}\text { Yes; inferior } \\
\text { STEMI } \\
\text { treated } \\
\text { with PCI }\end{array}$ & $\begin{array}{l}\text { Left femoral; } \\
\text { 55-cm Palindrome } \\
\text { catheter; } 106 \text { days }\end{array}$ & Sepsis & $\begin{array}{l}\text { CoNS } \\
\text { (Staphylococcus } \\
\text { caprae) }\end{array}$ & 10.4 & 6 & 35 & $20 \times 6$ & No & $\begin{array}{l}\text { RA free wall; } \\
\text { some mobile } \\
\text { extensions }\end{array}$ \\
\hline 14 & $\begin{array}{l}\text { 54/ } \\
\text { Female }\end{array}$ & 1 & Yes & Not known & $\begin{array}{l}\text { Right IJ; } 19-\mathrm{cm} \\
\text { Palindrome } \\
\text { catheter; } 6 \text { days }\end{array}$ & Sepsis & MSSA & 6.7 & 8 & 55 & $18 \times 12$ & No & $\begin{array}{l}\text { RA wall near } \\
\text { junction } \\
\text { with IVC; } \\
\text { some mobile } \\
\text { extensions }\end{array}$ \\
\hline
\end{tabular}

DM: diabetes mellitus; CABG: coronary artery bypass grafting; CoNS: coagulase-negative Staphylococcus; echo: echocardiography; Hb: haemoglobin; IHD: ischaemic heart disease; IJ: internal jugular; IVC: inferior vena cava; LVEF: left ventricular ejection fraction; MPI: myocardial perfusion imaging; MRSA: methicillin-resistant Staphylococcus aureus; MSSA: methicillin-susceptible Staphylococcus aureus; NSTEMI: non-ST-segment elevation myocardial infarction; PCI: percutaneous coronary intervention; RA: right atrium; STEMI: ST-segment elevation myocardial infarction 
Table II. Patient outcomes stratified by management strategy.

\begin{tabular}{|c|c|c|c|c|c|}
\hline Management & $\begin{array}{l}\text { Patient } \\
\text { no. }\end{array}$ & $\begin{array}{l}\text { Time from initial blood } \\
\text { culture to catheter } \\
\text { removal (day) }\end{array}$ & $\begin{array}{l}\text { Surgical procedure; } \\
\text { postoperative course }\end{array}$ & $\begin{array}{l}\text { Follow-up } \\
\text { echocardiography }\end{array}$ & $\begin{array}{l}\text { Mortality at } 180 \text { days } \\
\text { from echocardiographic } \\
\text { diagnosis }\end{array}$ \\
\hline \multirow{6}{*}{$\begin{array}{l}\text { Catheter removal } \\
\text { only }\end{array}$} & 2 & 7 & - & Nil & No \\
\hline & 3 & 5 & - & $\begin{array}{l}39 \text { days: } \\
\text { thrombus } 9 \times 8 \mathrm{~mm}\end{array}$ & No \\
\hline & 4 & 6 & - & Nil & No \\
\hline & 5 & 4 & - & Nil & Yes; Day 34 \\
\hline & 6 & 4 & - & $\begin{array}{l}48 \text { days: no } \\
\text { thrombus }\end{array}$ & No \\
\hline & 8 & 0 & - & Nil & Yes; Day 14 \\
\hline $\begin{array}{l}\text { Catheter } \\
\text { removal and } \\
\text { anticoagulation }\end{array}$ & 13 & 0 & - & Nil & No \\
\hline \multirow{2}{*}{$\begin{array}{l}\text { Anticoagulation } \\
\text { without catheter } \\
\text { removal }\end{array}$} & 10 & - & - & Nil & Yes; Day 16 \\
\hline & 11 & - & - & $\begin{array}{l}70 \text { days: no change } \\
\text { in thrombus size }\end{array}$ & No \\
\hline \multirow[t]{3}{*}{$\begin{array}{l}\text { Catheter removal } \\
\text { and surgery }\end{array}$} & 7 & 1 & $\begin{array}{l}\text { Thrombus removal and CABG; } \\
\text { postoperative pulmonary } \\
\text { embolism, nosocomial } \\
\text { pneumonia, septic shock }\end{array}$ & Nil & $\begin{array}{l}\text { Yes; Day } 51 \\
\text { POD } 32\end{array}$ \\
\hline & 9 & 0 & $\begin{array}{l}\text { Thrombus removal; } \\
\text { postoperative cardiac } \\
\text { tamponade requiring } \\
\text { resternotomy, ARDS and } \\
\text { nosocomial pneumonia } \\
\text { requiring ECMO }\end{array}$ & Nil & $\begin{array}{l}\text { Yes; Day } 49 \\
\text { POD } 36\end{array}$ \\
\hline & 12 & 4 & $\begin{array}{l}\text { Thrombus removal and CABG; } \\
\text { cardiac arrest POD 5, wound } \\
\text { infection requiring multiple } \\
\text { sternotomies, repeated } \\
\text { episodes of nosocomial sepsis, } \\
\text { prolonged hospitalisation } \\
\text { beyond } 6 \text { months }\end{array}$ & Nil & No \\
\hline
\end{tabular}

ARDS: acute respiratory distress syndrome; CABG: coronary artery bypass grafting; ECMO: extracorporeal membrane oxygenation; POD: postoperative day

dislodgement and subsequent pulmonary embolism, there are published recommendations to keep the catheter in situ initially and only attempt removal when therapeutic anticoagulation has been achieved, particularly for thrombi that are large, mobile or adherent to the catheter tip. ${ }^{(11,12)}$ In our series, this strategy was employed in Patient 10. However, haemodynamically significant pulmonary embolism despite therapeutic anticoagulation has also been reported post catheter removal. ${ }^{(2)}$ Interestingly, none of the patients in our series developed clinical pulmonary embolism, despite the majority having catheter removal during the index admission. There are three possible explanations for this observation. Firstly, our patients had relatively small thrombi that may not have been of sufficient size to cause clinically significant pulmonary embolism, although even catheters with large $(5.5 \mathrm{~cm})$ adherent thrombus have reportedly been safely removed without anticoagulation. ${ }^{(17)}$ Secondly, the location of the thrombi may influence the risk of pulmonary embolism. Among the cases with catheter removal, only two had thrombi adherent to the catheter at the time of echocardiography. The thrombi in the remaining cases were mostly attached to the RA free wall, which may result in greater stability as opposed to being on the catheter or at a position closer to the tricuspid valve. Finally, the mobility of the thrombi may also play a role. Half of the thrombi seen were assessed to be non-mobile, with most of the remaining mobile thrombi having only small mobile extensions, which may not result in symptoms even if embolism occurs. These multiple factors contribute to the difficulty in predicting embolic risk and therefore, decisions regarding catheter preservation or removal should be individualised.

Anticoagulation with vitamin $\mathrm{K}$ antagonists with a target international normalised ratio (INR) of 2.0-3.0 for six months or until thrombus resolution has been recommended for CRAT. ${ }^{(11)}$ However, only a minority of patients in our series received anticoagulation. Although we were unable to trace the reasons for not initiating anticoagulation in most patients, the most commonly documented reason for withholding anticoagulation was anaemia. 
Table III. Patient characteristics and mortality at 180 days.

\begin{tabular}{|llll|}
\hline Patient characteristic & \multicolumn{3}{c|}{ Median (range)/no. (\%)/mean \pm SD } \\
\cline { 2 - 4 } & All cases ( $\mathbf{n}=\mathbf{1 4})$ & Survivors ( $\mathbf{n} \mathbf{8})$ & Non-survivors (n= 6) \\
\hline Age (yr) & $59(47-88)$ & $59(53-88)$ & $56.5(47-67)$ \\
\hline Male gender & $11(78.6)$ & $6(75.0)$ & $5(83.3)$ \\
\hline Diabetes mellitus & $13(92.9)$ & $7(87.5)$ & $6(100)$ \\
\hline Known ischaemic heart disease & $6(42.9)$ & $4(50.0)$ & $2(33.3)$ \\
\hline Previous myocardial infarction & $3(21.4)$ & $2(25.0)$ & $1(16.7)$ \\
\hline Duration of dialysis (yr) & $2(1-5)$ & $1.5(1-5)$ & $2(1-2)$ \\
\hline Haemoglobin level on admission (g/dL) & $9.5 \pm 1.8$ & $9.3 \pm 2.0$ & $9.8 \pm 1.5$ \\
\hline Time from catheter insertion to 1st positive blood culture (day) & $92.0(1-424)$ & $105.5(1-393)$ & $28.5(5-424)$ \\
\hline Staphylococcus aureus infection & $7(50.0)$ & $2(25.0)$ & $5(83.3)$ \\
\hline Methicillin-resistant Staphylococcus aureus infection & $2(14.3)$ & $0(0)$ & $2(33.3)$ \\
\hline Time between admission to echocardiography (day) & $6.1 \pm 1.5$ & $6.4 \pm 1.1$ & $5.8 \pm 1.7$ \\
\hline Largest thrombus dimension (mm) & $23.4 \pm 7.8$ & $25.5 \pm 5.8$ & $21.8 \pm 8.6$ \\
\hline Thrombus with mobile components & $6(42.9)$ & $4(50.0)$ & $3(50.0)$ \\
\hline Left ventricular ejection fraction (\%) & $50.0(20-60)$ & $47.5(20-55)$ & $60.0(20-60)$ \\
\hline Catheter present at time of echocardiography & $7(50.0)$ & $5(62.5)$ & $2(33.3)$ \\
\hline Thrombus attached to catheter & $4(28.6)$ & $2(25.0)$ & $2(33.3)$ \\
\hline Catheter removal & $12(85.7)$ & $7(87.5)$ & $5(83.3)$ \\
\hline Anticoagulation & $4(28.5)$ & $3(37.5)$ & $1(20.0)$ \\
\hline Surgery & $3(21.4)$ & $1(12.5)$ & $2(33.3)$ \\
\hline
\end{tabular}

Indeed, ESRF results in haemostatic alterations that predispose the patient to bleeding. ${ }^{(18)}$ Observational data extrapolated from the use of warfarin in ESRF patients with atrial fibrillation is conflicting, with some studies demonstrating increased harm from anticoagulation. ${ }^{(18)}$ In light of these concerns, the Kidney Disease: Improving Global Outcomes (KDIGO) consensus statement discourages routine oral anticoagulation in dialysis patients with atrial fibrillation. ${ }^{(19)}$ These concerns regarding bleeding risk, together with the high incidence of anaemia, could explain why only a minority in our series received anticoagulation. Despite this, three out of four anticoagulated patients - including two with catheter removal - had favourable outcomes. This observation is consistent with a previously published case series in which seven out of ten cases of CRAT either resolved or reduced in size with anticoagulation at an INR target of 2.5-3.0, with no reports of bleeding complications. ${ }^{(5)}$ However, anticoagulation, when used alone in our patients, appears to be less beneficial in terms of reducing mortality or thrombus burden. A possible reason is that the catheter continues to serve as a nidus for both infection and thrombus formation, thus negating the effects of antibiotic and anticoagulation therapy.

The role of surgical thrombectomy is not well defined in the literature. A large series consisting of both infective and non-infective haemodialysis CRAT did not show a mortality difference between surgery and anticoagulation. ${ }^{(12)}$ Other authors have reported good surgical outcomes, ${ }^{(16)}$ including the use of minimally invasive surgery. ${ }^{(17)}$ There are several published recommendations supporting the use of surgery for large and/or mobile thrombi. ${ }^{(4,15,16)}$ We have, however, demonstrated very poor surgical outcomes in all three operated cases in our series.
This is unlikely to be due to poor patient selection, as all three had under 10\% surgical risk estimated using EUROSCORE II and documented clearance of bacteraemia preoperatively. Notably, the EUROSCORE II already includes renal impairment, urgency of procedure, presence of endocarditis and number of procedures (thrombectomy with or without bypass) as variables in the estimation of risk. ${ }^{(7)}$ This may suggest that validated risk calculators for general cardiac surgery may not necessarily apply to patients with infective haemodialysis CRAT, even when patient-specific risk factors are taken into consideration.

Haemodialysis CRAT is associated with significant mortality. A large series of both infective and non-infective CRAT cases encountered up to the year 2010 demonstrated a mortality of $18 \%$. ${ }^{(12)}$ Negulescu et al demonstrated that mortality was 33\% in infective cases compared to $14 \%$ in non-infective CRAT. ${ }^{(4)}$ Indeed, the mortality rate in our series approximates that of a more recent study of IE in haemodialysis patients, which showed an inpatient mortality of $41 \%$, with an additional $14 \%$ mortality within the first year. ${ }^{(20)}$ The high mortality rate among haemodialysis CRAT cases is not surprising given that the known adverse prognostic factors for IE in haemodialysis patients include diabetes mellitus and rightsided $\mathrm{IE},{ }^{(21)}$ which are present in almost all of our patients. Our small patient numbers preclude us from evaluating the variables in Table III statistically for any significant association with mortality at 180 days. However, we have observed a very high number of cases with Staphylococcus aureus bacteraemia among the nonsurvivors. Although this finding remains hypothesis-generating, it is consistent with other publications demonstrating the high case fatality rate of Staphylococcus aureus bacteraemia among ESRF patients, particularly those on haemodialysis. ${ }^{(9)}$ 
Our study has several limitations. Firstly, the retrospective study design limits our ability to draw comparative conclusions about the various treatment modalities and is highly dependent on the accuracy of the reviewed electronic medical records. The relationship between treatment and thrombus resolution cannot be confidently made, as not all patients had repeat imaging at a pre-determined time interval. Secondly, we were only able to describe a small number of patients in this case series given the rarity of this condition, although to our knowledge, this is the largest case series of infective haemodialysis CRAT reported in this decade. Finally, our cases were collected from a single tertiary medical institution and may not necessarily be universally representative of all patients with infective haemodialysis CRAT.

In summary, our study is the largest case series evaluating the management and outcomes of infective haemodialysisrelated CRAT in the current medical literature. Contrary to published recommendations, only a small number of patients received anticoagulation, possibly due to concerns of anaemia and bleeding risk. Most of our anticoagulated patients had favourable outcomes, particularly for those who had catheter removal as well. Surgical outcomes remain poor despite current techniques and should be reserved for selected cases. The overall mortality for infective haemodialysis CRAT remains high despite contemporary management. Although our small series precludes us from identifying patient or treatment variables that are significantly associated with mortality, we have observed a very high proportion of Staphylococcus aureus infection among the non-survivors. Data from further prospective studies is needed to verify this finding and guide us on the optimal management strategy for this group of patients.

\section{REFERENCES}

1. Vascular Access 2006 Work Group. Clinical practice guidelines for vascular access. Am J Kidney Dis 2006; 48 Suppl 1:S176-247.

2. Asmarats L, Fernández-Palomeque C, Martínez-Riutort JM, Bethencourt A Right atrial thrombosis associated with hemodialysis catheter: first description of recurrence in a poorly understood problem. J Thromb Thrombolysis 2015; 39:254-7.

3. Powe NR, Jaar B, Furth SL, Hermann J, Briggs W. Septicemia in dialysis patients: incidence, risk factors, and prognosis. Kidney Int 1999; 55:1081-90.

4. Negulescu O, Coco M, Croll J, Mokrzycki MH. Large atrial thrombus formation associated with tunneled cuffed hemodialysis catheters. Clin Nephrol 2003; 59:40-6.

5. Shah A, Murray M, Nzerue C. Right atrial thrombi complicating use of central venous catheters in hemodialysis. Int J Artif Organs 2004; 27:772-8.

6. Nashef SA, Roques F, Sharples LD, et al. EuroSCORE II. Eur J Cardiothorac Surg 2012; 41:734-45.

7. Forauer AR, Theoharis $\mathrm{C}$. Histologic changes in the human vein wall adjacent to indwelling central venous catheters. J Vasc Interv Radiol 2003; 14(9 Pt 1):1163-8.

8. Kato S, Chmielewski $\mathrm{M}$, Honda $\mathrm{H}$, et al. Aspects of immune dysfunction in end-stage renal disease. Clin J Am Soc Nephrol 2008; 3:1526-33.

9. Nielsen LH, Jensen-Fangel S, Benfield T, et al. Risk and prognosis of Staphylococcus aureus bacteremia among individuals with and without endstage renal disease: a Danish, population-based cohort study. BMC Infect Dis 2015; 15:6.

10. Zhang J, Burr RA, Sheth HS, Piraino B. Organism-specific bacteremia by hemodialysis access. Clin Nephrol 2016; 86:141-6.

11. Stavroulopoulos A, Aresti V, Zounis C. Right atrial thrombi complicating haemodialysis catheters. A meta-analysis of reported cases and a proposal of a management algorithm. Nephrol Dial Transplant 2012; 27:2936-44.

12. Sontineni SP, White $M$, Singh $S$, et al. Thrombectomy reduces the systemic complications in device-related right atrial septic thrombosis. Can J Cardiol 2009; 25:e36-41.

13. Thakar S, Janga KC, Tolchinsky T, et al. Superior vena cava and right atrium wall infective endocarditis in patients receiving hemodialysis. Heart Lung 2012; 41:301-7.

14. Weinberg L, Zia F, Tay S, Roubos N. Haemodialysis catheter-related giant right atrial and superior cava thrombi. BMJ Case Rep 2015; 2015:bcr2015209350.

15. Mach L, Ondruskova O, Nemec P, Orban M. Massive catheter-related thrombosis of vena cava superior protruding into the right atrium in a hemodialysis patient. Hemodial Int 2015; 19:E10-3.

16. Chan J, Kumar J, Cheng A, Yap CH, Zhang XB. Right atrial mass associated with a dialysis catheter. J Card Surg 2012; 27:362.

17. Lin Cl, Chen HH, Chen YC, Wu CJ. Catheter-related atrial thrombus resolved after catheter removal in a patient on hemodialysis. South Med J 2008; 101:662-3.

18. Black-Maier E, Piccini JP. Oral anticoagulation in end-stage renal disease and atrial fibrillation: is it time to just say no to drugs? Heart 2017; 103:807-8.

19. Herzog CA, Asinger RW, Berger AK, et al. Cardiovascular disease in chronic kidney disease. A clinical update from Kidney Disease: Improving Global Outcomes (KDIGO). Kidney Int 2011; 80:572-86.

20. Ramos-Martínez A, Roque F, Fariñas MC, et al. Prognostic factors of infective endocarditis in patients on hemodialysis: a case series from a National Multicenter Registry. Int J Cardiol 2017; 241:295-301.

21. McCarthy JT, Steckelberg JM. Infective endocarditis in patients receiving longterm hemodialysis. Mayo Clin Proc 2000; 75:1008-14. 